about $10 \mu \mathrm{g}$, enough for more than 100 PCRs. No degradation was found after incubation at $37^{\circ} \mathrm{C}$ for one month (figure), indicating the long term stability of the sample at $4^{\circ} \mathrm{C}$. The clotted blood before DNA extraction can be stored at $-20^{\circ} \mathrm{C}$ in the tube used for serum separation for up to a month, and can be transferred on dry ice to other laboratories.

\section{Discussion}

The salt/chloroform method is better than the use of phenol, because it avoids having to use a dangerous solvent. However, unlike the procedure described before, ${ }^{4}$ neither absolute ethanol nor propanol are recommended for DNA precipitation because it also precipitates salts and blood pigments, which inhibit poly- merases and restriction enzymes. This method is simple, rapid, economical, and within the scope of most clinical laboratories. It facilitates the collection of many samples for studies of genetic disorders because it uses normally discarded blood clots, and is widely applicable to medical, genetic, epidemiological, and forensic studies.

This work was supported in part by a Grant-in-Aid for Scientific Research from the Ministry of Education, Science and Culture of Japan.

1 Innis MA, Gelfand DH, Sninsky JJ, White TJ. PCR protocol. San Diego: Academic Press, 1990.

2 Panaccio M, Lew A. PCR based diagnosis in the presence of $8 \%$ (v/v) blood. Nucleic Acids Res 1991;19:1151.

3 McCusker J, Dawson MT, Noone D, Gannon F, Smith T. Improved method for direct PCR amplification from whole blood. Nucleic Acid Res 1992;20:6747.

4 Müllenbach $R$, Logoda PJL Welter C An efficient saltchloroform extraction of DNA from blood and tissues Trends Genet 1989;5:391.

\title{
Comparison of formalin and Bouin's reagent for fixation of coagulase negative staphylococcal biofilm
}

\author{
M H Wilcox
}

\begin{abstract}
Methodological modifications, particularly the use of different fixatives, may account for discrepancies between studies of the relation between virulence and biofilm production in vitro by isolates of coagulase negative staphylococci. The efficacy of formalin and Bouin's reagent for fixing coagulase negative staphylococcal biofilms in a microtitre tray assay was compared. The optical density of stained adherent growth by three strains was reduced by an average of $20 \%$ following fixation with $10 \%$ formaldehyde compared with Bouin's reagent. This difference seemed to be mainly because of increased background staining and blackening of the biofilm when Bouin's reagent was used. Formalin fixation was also effective at identifying early and late biofilm production in adherence growth kinetic experiments with 10 coagulase negative staphylococcal clinical isolates.
\end{abstract}

(f Clin Pathol 1994;47:1044-1046)

Clinical Microbiology and Public Health Laboratory, Level 6, Addenbrooke's Hospital, Cambridge, CB2 2QW M H Wilcox

Correspondence to: Dr M H Wilcox

Accepted for publication 19 April 1994

Coagulase negative staphylococci adhere to and accumulate on polymer surfaces by producing extracellular slime, forming biofilms. Although authors have frequently referred to the in vitro measurement of coagulase negative staphylococcal slime production, the most commonly used methods ${ }^{1}$ primarily examine surface accumulation of bacterial cells, rather than slime per se. ${ }^{2}$ The term "biofilm" has been used here as a compromise, accepting that cell accumulation is the main parameter under investigation. Many groups have examined biofilm formation by coagulase negative staphylococci in vitro to determine whether clinical isolates associated with infection of medical devices are more adherent than control strains, with conflicting results. ${ }^{13-8}$ Methodological differences in the commonly used microtitre tray assay of adherent growth are possible causes of some of these discrepant results. ${ }^{1-578}$ In particular, methods used to fix the coagulase negative staphylococcal biofilm to the bottom of the wells in the microtitre tray after washing may not be equally effective. ${ }^{9}$ The original report of the microtitre tray assay described the use of Bouin's reagent as a fixative. ${ }^{1}$ This reagent, however, is potentially explosive, relatively expensive, and less readily available than alternatives such as formalin and glutaraldehyde, which have been used as fixatives in some cases. ${ }^{8} 10$ This study compares the efficacy of formalin and Bouin's reagent for the fixation of coagulase negative staphylococcal biofilms in vitro.

\section{Method}

Twelve coagulase negative staphylococcal clinical isolates were obtained from patients with peritonitis undergoing continuous 
ambulatory peritoneal dialysis (CAPD). These were identified by API Staph (API, Basingstoke, UK) and included 10 strains of Staphylococcus epidermidis (s1, s2, s3, s8, s31, s34, s49, s59, s900, and s902), one of Staphylococcus warneri (s79), and one of Staphylococcus haemolyticus (s907). A well described coagulase negative staphylococcal strain which is positive in the tube and microtitre tray adherence assays, $S$ epidermidis ATCC 35984, was used as a control. ${ }^{1}$ Strains $\mathrm{s} 1, \mathrm{~s} 2$, and $\mathrm{s} 3$ were chosen at random from a collection of CAPD isolates, and the remainder of the clinical isolates were known adherence positive strains, as described before. ${ }^{10}$ Coagulase negative staphylococcal were stored in horse serum at $-70^{\circ} \mathrm{C}$ and were maintained by subculture on blood agar.

The microtitre tray adherence assay has been described previously. ${ }^{1}$ Briefly, an inoculum equivalent to a final concentration of $10^{6}$ colony forming units $/ \mathrm{ml}$ was prepared from an overnight culture in tryptone soya broth (TSB; Oxoid, Hampshire, UK). Polystyrene microtitre trays with flat bottomed wells (M29A, Dynatech Laboratories, Sussex, UK) were used, with a final well volume of $250 \mu \mathrm{l}$. Following incubation in TSB in air at $37^{\circ} \mathrm{C}$, well contents were removed and non-adherent bacteria displaced by washing three times with saline. Fixation consisted of adding $75 \mu \mathrm{l}$ of either Bouin's reagent $(75 \mathrm{ml}$ picric acid, $25 \mathrm{ml}$ formalin, and $5 \mathrm{ml}$ glacial acetic acid) or $10 \% \mathrm{v} / \mathrm{v}$ formaldehyde $(25 \% \mathrm{v} / \mathrm{v}$ formalin; $\mathrm{BDH}$, Poole, Dorset, UK) to each well for 30 minutes. Fixed biofilms were stained with crystal violet and, after further washing and air drying, the optical density of adherent bacterial growth was measured using a microtitre tray spectrophotometer (SLT 210, Kontron Analytical Instruments, Zurich, Switzerland) at an absorbance of 546 or 600 $\mathrm{nm}$. Background staining was assessed in control wells inoculated with sterile TSB.

Biofilm formation by strains $s 1, s 2$, and $s 3$, following incubation for 24 hours, was compared in the microtitre tray adherence assay following fixation with either Bouin's reagent

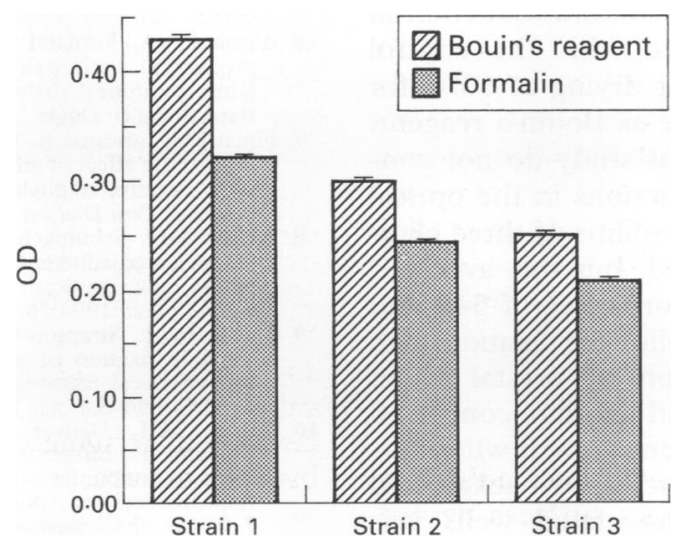

Figure 1 Comparison of biofilm formation by three $S$ epidermidis strains following fixation with Bouin's reagent or $10 \%$ formaldehyde, measured as the optical density (OD) of stained bacteria at an absorbance of $546 \mathrm{~nm}$. or $10 \%$ formaldehyde. For each method of fixation, strains were cultured in eight wells in each of three different microtitre trays, and mean and SEM values for the 24 separate readings calculated. In a separate series of experiments the kinetics of coagulase negative staphylococcal adherent growth by 10 strains was examined to determine whether fixation with $10 \%$ formaldehyde alone permitted identification of both early and late biofilm formation. In duplicate experiments strains were inoculated into eight wells in each of seven microtitre trays. Adherent growth was measured in each tray after incubation periods of two, four, six, eight, 18, 24, and 48 hours and mean optical densities were calculated.

\section{Results}

The inter- and intratray coefficients of variation of adherent growth for a control coagulase negative staphylococcal strain were both consistently less than $10 \%$. Biofilms were successfully fixed by either Bouin's reagent or $10 \%$ formaldehyde (fig 1). Biofilm was macroscopically visible as black colonies following fixation with Bouin's reagent and as violet coloured adherent growth when $10 \%$ formaldehyde was used. The optical densities of stained biofilms were reduced by 25,18 , and $17 \%$ with strains $s 1, s 2$, and $s 3$, respec-

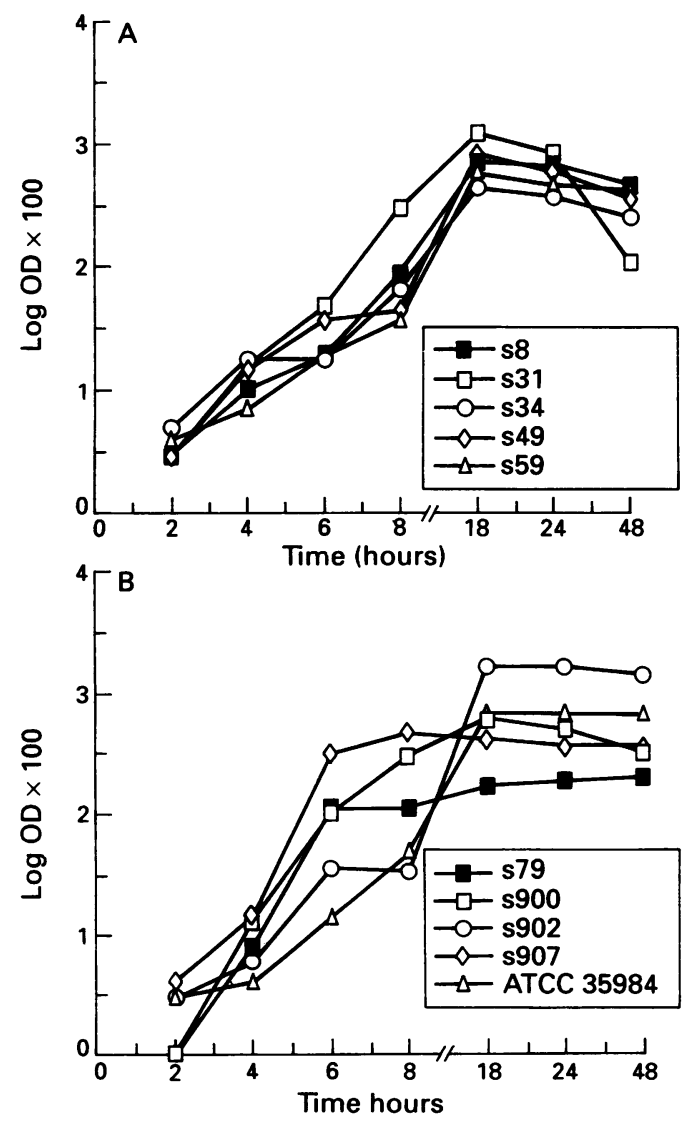

Figure 2 (A) Kinetics of biofilm formation by 10 coagulase negative staphylococci following fixation with $10 \%$ formaldehyde, measured as the optical density (OD) of stained bacteria at an absorbance of $546 \mathrm{~nm}$. (B) Relatively early biofilm formation was observed for $s 79$ and $s 907$. 
tively, following fixation with $10 \%$ formaldehyde compared with Bouin's reagent. Corresponding reductions in optical density at an absorbance of $600 \mathrm{~nm}$ were 14, 9, and $8 \%$, respectively (data not shown). Optical densities of control wells, indicating the level of background staining, were $30 \%$ lower, on average (the mean reduction in optical density was 0.7 at $546 \mathrm{~nm}$ ), following fixation with $10 \%$ formaldehyde compared with Bouin's reagent.

The kinetics of biofilm formation were identified clearly and noticeable interstrain variations observed following fixation with $10 \%$ formaldehyde (fig 2 ). The majority of strains achieved maximal biofilm formation after incubation for 18 hours (fig 2A); increases in optical density were not observed after 18 hours. Close to maximal adherent growth was observed with coagulase negative staphylococcal strains s79 and s907 after incubation for six hours (fig 2B). Viable counts of planktonic bacteria increased at least 10 -fold after between eight and 18 hours of incubation (data not shown).

\section{Discussion}

The quantitative microtitre tray assay of coagulase negative staphylococcal biofilm formation is clearly superior in terms of discriminatory power and reproducibility to the qualitative tube test. ${ }^{14}$ There is disagreement in the literature over whether there is an association between the virulence of coagulase negative staphylococci and biofilm production in vitro. Substitution of Bouin's reagent with either formalin or other fixatives is a possible cause of such discrepant results in the microtitre tray assay. Conflicting results, however, have been obtained using both the tube test, ${ }^{46}$ which does not involve a stage to fix bacterial adherent growth to the polymer surface, and the microtitre tray assay. ${ }^{13-578}$

Baldassarri et al compared 12 different methods of fixation of a bacterial biofilm using a single strain, a defined clone of the control $S$ epidermidis strain ATCC $35984 .{ }^{9}$ They found that neither $2.5 \%$ nor $10 \%$ formaldehyde adequately fixed the staphylococcal biofilm, with a severalfold reduction in optical density compared with the control biofilm, and that only air drying at $60^{\circ} \mathrm{C}$ for one hour was as effective as Bouin's reagent. The results of the present study do not confirm these findings. Reductions in the optical densities of the stained biofilms of three clinical isolates were observed, but this averaged only 20 and $10 \%$ at absorbances of 546 and $600 \mathrm{~nm}$, respectively. Following fixation with Bouin's reagent, addition of crystal violet results in a black stained biofilm compared with violet stained adherent growth when formalin fixation is used. Increased background staining of control uninoculated wells was evident when Bouin's reagent was used. Furthermore, in contrast to the observations of Baldassarri et al, biofilm fixation with formalin enabled identification of early and late adherent growth for all 10 coagulase negative staphylococcal strains studied (including $S$ epidermidis ATCC 35984$).{ }^{9}$ Biofilm formation by two strains was notably more rapid and the importance of these observations warrants further study. Clonal variation of $S$ epidermidis ATCC 35984 may account for the different results obtained in the two studies. In the author's experience the washing procedure is the most difficult part of the assay to standardise, but this is rarely regarded as a source of error.

Formalin is widely available, inexpensive, and gives reproducible results when used as a fixative in the microtitre tray adherence assay. Air drying at $60^{\circ} \mathrm{C}$ has been suggested as an alternative method of fixation, but hot air ovens are not always available. ${ }^{9}$ If a modification of the microtitre tray adherence assay is used, ${ }^{7}{ }^{10}$ consisting of silicone rubber discs fixed by grease to the bottom of the wells, prolonged drying leads to detachment of the discs and, therefore, to errors during measurement of the optical density of stained biofilm (data not shown). This modified method is useful for the study of biofilm formation on clinically relevant polymers such as silicone rubber, which is often very different compared with the polystyrene used in the manufacture of microtitre trays. ${ }^{810}$

1 Christensen GD, Simpson WA, Younger JJ, Baddour LM Barrett FF, Melton DM, et al. Adherence of coagulasenegative staphylococci to plastic tissue culture plates: quantitative model for the adherence of staphylococci to medical devices. F Clin Microbiol 1985;22:996-1006.

2 Hussain M, Wilcox MH, White PJ. The slime of coagulase-negative staphylococci: biochemistry and relation to adherence. FEMS Microbiol Rev 1993;104:191-208.

3 Baddour LM, Smalley DL, Kraus AP, Lamoreauz WJ, Christensen GD. Comparison of microbiologic characChristensen GD. Comparison of microbiologic characeristics of pathogenic and saprephys 作 1986;5:197-205.

4 Deighton MA, Balkau B. Adherence measured by microtitre assay as a virulence marker for Staphylococcus epidermidis infections. F Clin Microbiol 1990;28:2442-7.

5 Younger JJ, Christensen GD, Bartley DL, Simmons JCH Barrett FF. Coagulase-negative staphylococci isolated from cerebrospinal fluid shunts: importance of slime production, species identification and shunt removal to clinical outcome. $\mathcal{F}$ Infect Dis 1987;156:548-54.

6 Alexander A, Rimland D. Lack of correlation of slime production with pathogenicity in continuous ambuproduct peritoneal dialysis caused by coagulase-negative latory peritoneal dialysis caused by coagulase-negative staphylococci. Diagn Microbiol Infect Dis 1987;8:215-20. inch RG, Edwards R, Filik R, Wilcox MH. CAPD peritonitis: the effect of antibiotic on the adherence of coagulase-negative staphylococci to silicone rubber cathete material. Perit Dial Int 1989;9:103-5.

8 Wilcox MH, Schumacher-Perdreau F. Lack of evidence for increased adherent growth in broth or human serum of clinically significant coagulase-negative staphylococci. f Hosp Infect 1994;26:239-50.

9 Baldassarri L, Simpson WA, Donelli G, Christensen GD Variable fixation of staphylococcal slime by differen histochemical fixatives. Eur $\mathcal{F}$ Clin Microbiol Infect Di 1993;12:866-8.

10 Wilcox MH, Denyer SP, Finch RG, Smith DGE Williams P. Effects of carbon dioxide and sub-letha levels of antibiotics on adherence of coagulase-negative staphylococci to polystyrene and silicone rubber. f Antimicrob Chemother 1991;27:577-87. 